\title{
Big innovations in a small instrument: technical challenges in a new CCD system design for the Automated Patrol Telescope
}

\author{
Stan Miziarski.* ${ }^{\text {a }}$, Michael C. B. Ashley ${ }^{\mathrm{b}}$, Greg Smith, ${ }^{\mathrm{a}}$ Sam Barden ${ }^{\mathrm{a}}$, John Dawson ${ }^{\mathrm{a}}$, \\ Anthony Horton ${ }^{a}$, Will Saunders ${ }^{a}$, Jurek Brzeski ${ }^{a}$, Vladimir Churilov ${ }^{a}$, Urs Klauser ${ }^{a}$, \\ Lew Waller ${ }^{\mathrm{a}}$, Don Mayfield ${ }^{\mathrm{a}}$, David Correll ${ }^{\mathrm{a}}$, Andre Phillips ${ }^{\mathrm{b}}$, Denis Whittard ${ }^{\mathrm{a}}$ \\ ${ }^{a}$ Anglo-Australian Observatory, PO Box 196 Epping NSW 1710 Australia; \\ ${ }^{b}$ School of Physics, University of New South Wales, Sydney NSW 2052 Australia
}

\begin{abstract}
We describe the design of a new CCD system delivered to the Automated Patrol Telescope at Siding Springs NSW Australia operated by UNSW. A very fast beam (f/1) with a mosaic of two MITLL CCID-34 detectors placed only 1 $\mathrm{mm}$ behind the field flattener which also serves as the dewar window, have called for innovative engineering solutions. This paper describes the design and procedure of the field-flattener mounting, differential screw adjustable detector mount and dewar suspension on the external ring providing tip/tilt and focus adjustment.
\end{abstract}

Keywords: CCD, Schmidt telescope

\section{INTRODUCTION}

The Automated Patrol Telescope (APT) is a wide-field CCD imaging telescope, which is operated by the University of New South Wales at Siding Spring Observatory, Australia.

The optical design employed resembles that of a Schmidt camera, but uses a 3-element lens to achieve a wide, corrected field-of-view ${ }^{1}$. Telescope motion and operation of the CCD have been placed under computer control, allowing automated observations for long-term survey and monitoring projects. The APT has $0.5 \mathrm{~m}$ aperture f/1 optics which produce a 5 degree flat field, of which a $2 \times 3$ degree field was utilised by the CCD formerly installed. Imaging could be done either unfiltered or through $\mathrm{B}, \mathrm{V}, \mathrm{R}$ and I broad-band filters.

In common with most physical sciences, breakthroughs in astronomy are often the result of the construction of instruments that open up new regions of parameter space.

In astronomical optical imaging, the two of the most important parameters are the diameter of the telescope mirror, and the field-of-view that can be covered with charge-coupled device (CCD) detectors.

Due to fundamental device limitations, it is currently impractical to simultaneously have both large mirrors (e.g., $>4 \mathrm{~m}$ in diameter) and large fields of view (e.g., > 10 square degrees) that are fully populated with detectors. The world's largest telescopes (10m in diameter) have fields measured in hundredths of a square degree. An alternative solution is to sparsely populate a large field with optical fibres. This approach has been pioneered through technology developed at the Anglo-Australian Observatory (AAO), and is used on the $2 \mathrm{dF}$ instrument on the Anglo-Australian Telescope, and $6 \mathrm{dF}$ on the AAO Schmidt telescope. However, this solution is only suitable for spectroscopy.

The aim of this project was to equip a modest-aperture telescope (the $0.5 \mathrm{~m}$ Automated Patrol Telescope (APT) owned and operated by UNSW at Siding Spring Observatory) with a huge field-of-view (5.7×5.7 degrees).

Advanced Optical and Mechanical Technologies in Telescopes and Instrumentation, edited by Eli Atad-Ettedgui, Dietrich Lemke, Proc. of SPIE Vol. 7018, 70184G, (2008) · 0277-786X/08/\$18 · doi: 10.1117/12.788624 
This approach has only become possible in the last few years through advances in CCD technology and in the computers needed to process the resulting images. The AAO was contracted to design and build the new camera, and the associated mounting interface and robotic filter-changer.

The new camera will cover 5 times the area and with 3 times the quantum efficiency of the previously used camera. For many projects, this represents a 15 times increase in throughput. The individual pixels in the camera will be $1 / 5$ th the area, leading to substantial improvements in resolution, photometric precision, and dynamic range. Furthermore, the camera will use a $1 \mathrm{MHz}$ readout rate (fast for an astronomical CCD), and two video chains per CCD, allowing the entire image to be read in 9.4 seconds, thereby reducing dead-time and increasing observing efficiency. For comparison, typical CCD mosaic cameras (such as WFI on the AAT, or PFC on the $2.5 \mathrm{~m}$ INT telescope on La Palma) cover areas of $0.5 \times 0.5$ degrees $(<1 \%$ of the area of our camera), and take $\sim 2.5$ minutes to read out.

The new instrument occupies a region of parameter space that has previously only been explored with photographic plates on Schmidt telescopes. In contrast to photographic plates, which are highly nonlinear and have quantum efficiencies of a few percent, CCDs are almost ideal detectors, with $<0.1 \%$ nonlinearity and up to $90 \%$ quantum efficiency.

Our project was technically challenging due to the need to package a large collecting area into a camera with a small cross-sectional area (so that it does not obscure the telescope beam).

The $1 \mathrm{MHz}$ readout rate is also pushing the state-of-the-art in this field. Furthermore, the AAO needed to develop techniques in packaging CCD mosaics, which was a new area of expertise for them. It is for this reason that the AAO was willing to make an in-kind contribution of engineering time to this project.

This paper deals with mechanical engineering challenges called upon by extreme demands of the optical design and planned operational requirements of the APT. It concentrates on the methods and technologies used, with the aim of potentially being helpful to others in the design of instruments with similar challenges. The calculations have not been quoted, as they were of rather specific nature to this project.

\section{OPTICAL DESIGN CONSTRAINTS}

The APT is a small telescope. With a $0.5 \mathrm{~m}$ aperture, the detector has to occupy as small space as possible to prevent excessive vignetting. The target for the outside diameter of the dewar was set to $165 \mathrm{~mm}$.

The next challenge was that the optical beam filled a large fraction of the telescope tube. In the small amount of available volume we had to fit an automated four filter changer, as well as tip, tilt and focus adjustment. The shape of the optical beam begins as a circle (the corrector aperture) and morphs to a square (the CCD mosaic). This constrained the possible location of the filter changer.

For the tip tilt and focus adjustment we have chosen a spider and ring arrangement with three actuators. There was no room inside the telescope tube for this arrangement. We have used four inspection holes in the telescope tube to carry the spider vanes through and installed the ring and actuators outside the telescope tube.

Alignment of the mosaic of two MITLL CCID-34 detectors was another difficult task. The optical design called for 2 micron flatness tolerance, for which we had to find a novel solution. 


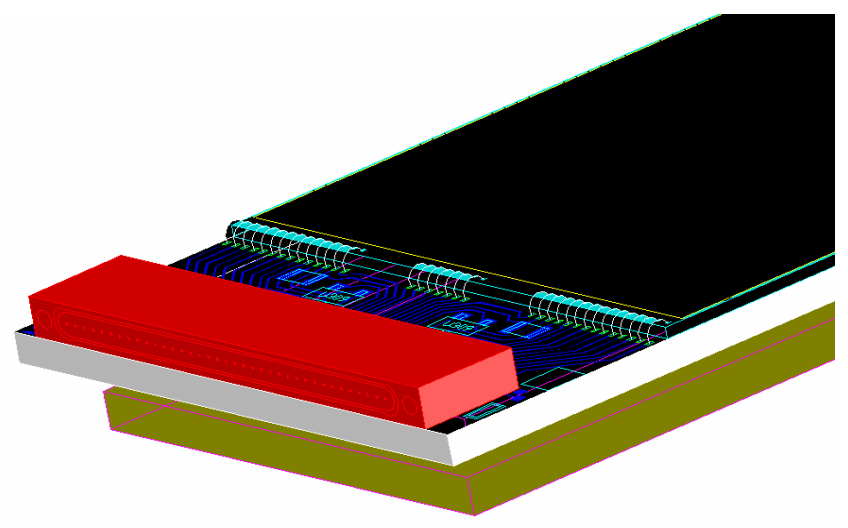

Fig. 1. Detail of the MITLL CCID-34 detector showing a nanoconnector and circularly looped gold wires.

There was a serious conflict between the MITLL CCID-34 design and the requirements of the optical design. The surface of the detector had to be exactly $1 \mathrm{~mm}$ from the face of the field flattener. As mechanical engineers, we could not negotiate any more than this from the optical designers. The difficulty was that the nanoconnector (red colored in the image Fig.1) was protruding $1.5 \mathrm{~mm}$ above the (black) face of the detector. We also had to be very careful about the fragile, circularly looped gold wires between the CCD and its nanoconnector. This called for designing a challenging way of mounting of the field flattener lens in the dewar lid and providing a recess in the cover, near the detector edge, to clear the nanoconnector. It left us with a very thin and narrow lip to seal the lens against.

\section{FILTER CHANGER}

The examination of space available inside the telescope tube (Fig.2) and clearing the light beam, uncovered four locations where filters and their actuators could be mounted. Two of them were in the area where mounting brackets could be attached to the telescope tube structural elements. The size of the filter made any attempt to mount the changer on the camera unrealistic.

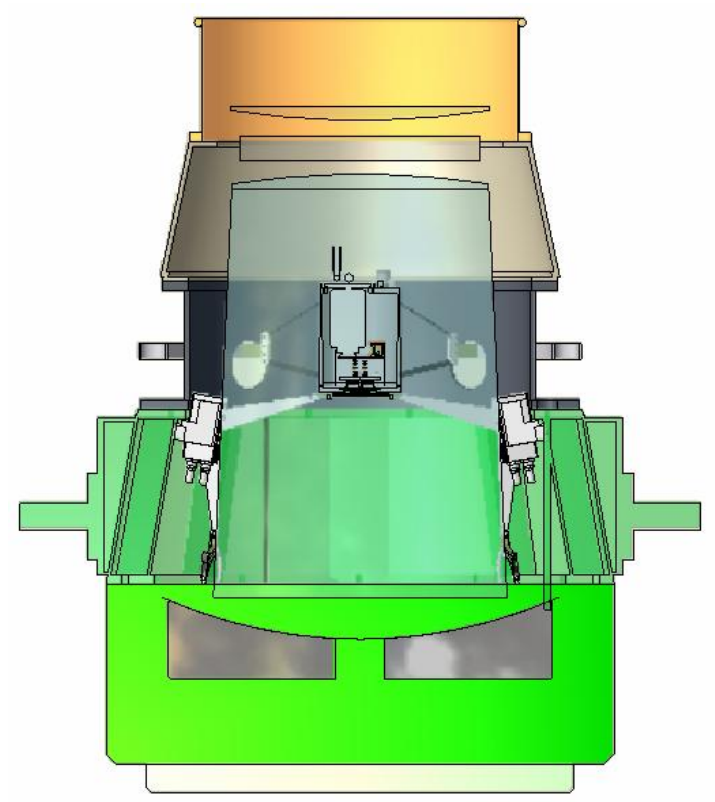

Fig.2. Section view of the APT showing the light beam and filter changers mounted on both sides. 
Mounting the filter changers independently of the dewar required some flexure in the filter-changer arms and filter frames, to allow repetitive precise location of the filters against the dewar window. This has been achieved by a variety of double gimbal mount with some freedom to slide in the arm length direction and a flex machined close to the tip of the arm. Quick but smooth motion of the filter arms was achieved by use of FESTO ${ }^{\circ}$ pneumatic semirotary actuators (ZP-JE-175803DRQD-25-90-YSRJ-A-AR-FW). There are interlocks in the electronic control of the changer preventing collision of the filters.

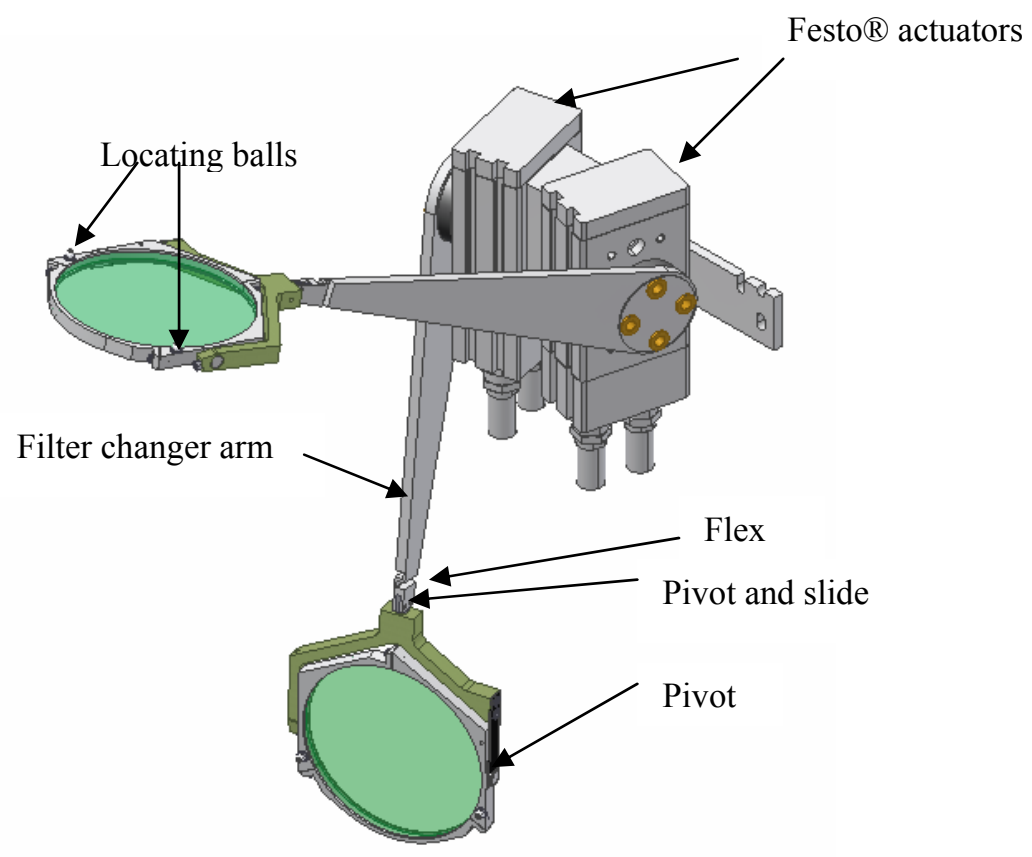

Fig 3. One of two filter changer subassemblies

Figure 3 shows the subassembly of two arms of the filter changer. The upper arm is shown in the engaged position, the other is retracted. The locating balls indicated in the Figure engage with corresponding close fit cavities (one circular and one elongated) in the dewar lid. Testing of the filter changer combined with the functional and static deflection test of the dewar mount has been performed on a specially designed rigid jig shown in Figure 4.

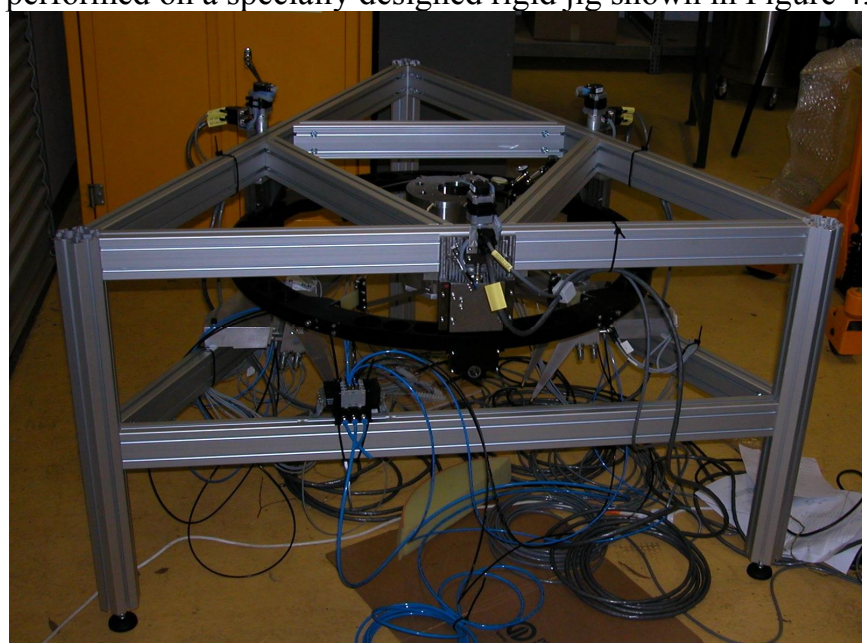

Fig. 4. The tip , tilt, focus drives and filter changer testing jig.

The tests were performed with the jig tilted from $0^{\circ}$ to $90^{\circ}$ to cover more than the range of motion of the telescope. 


\section{DEWAR MOUNT}

When designing the dewar mount, we had similar concerns about potentially obscuring the light beam as with the filter changer. We had to bring the actuators to the outside of the telescope, but did not want to machine new holes in the telescope tube if possible. W Fortunately, there were four suitable pre-existing holes of $\sim 100 \mathrm{~mm}$ diameter and we designed the dewar mounting ring and spider in a way that allowed the passage of the spider vanes through those holes with enough clearance for tip, tilt and focus adjustment.

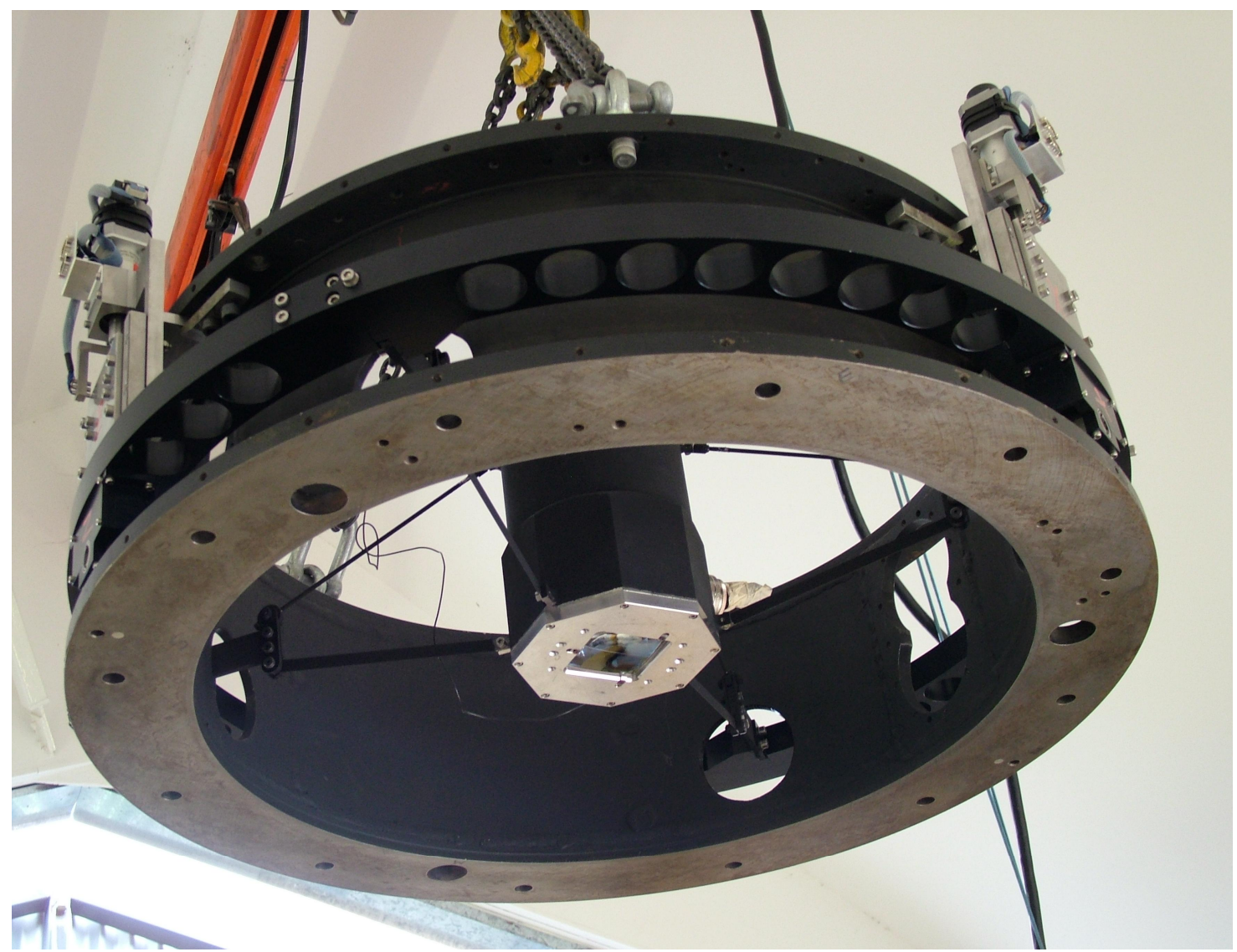

Fig. 5. Telescope tube with the dewar mounted, spider vanes passing though the existing holes in the telescope tube.

The dewar, ring and spider assembly is held in position by linear actuators from Digit ${ }^{\circ}$ stabilized with SKF® V slide linear bearings. Digit ${ }^{\circledR}$ actuators were driven by stepper motors with Quicksilver ${ }^{\circ}$ amplifiers. The low resistance tip/tilt is allowed by a combination of three sets of two flexes. The first flex is a specially designed figure A shaped member with three blades (see Figure 8). The other is a commercial Riverhawk ${ }^{\circledR}$ double ended flexural pivot bearing Visible in the bottom part of (Figure 7). The whole setup has been analysed in all relevant positions with FEA. Deflections between drive mounts and the dewar were measured on the test jig with the use of dial gauges and an electronic protractor. The test results confirmed our expectations from FEA. 


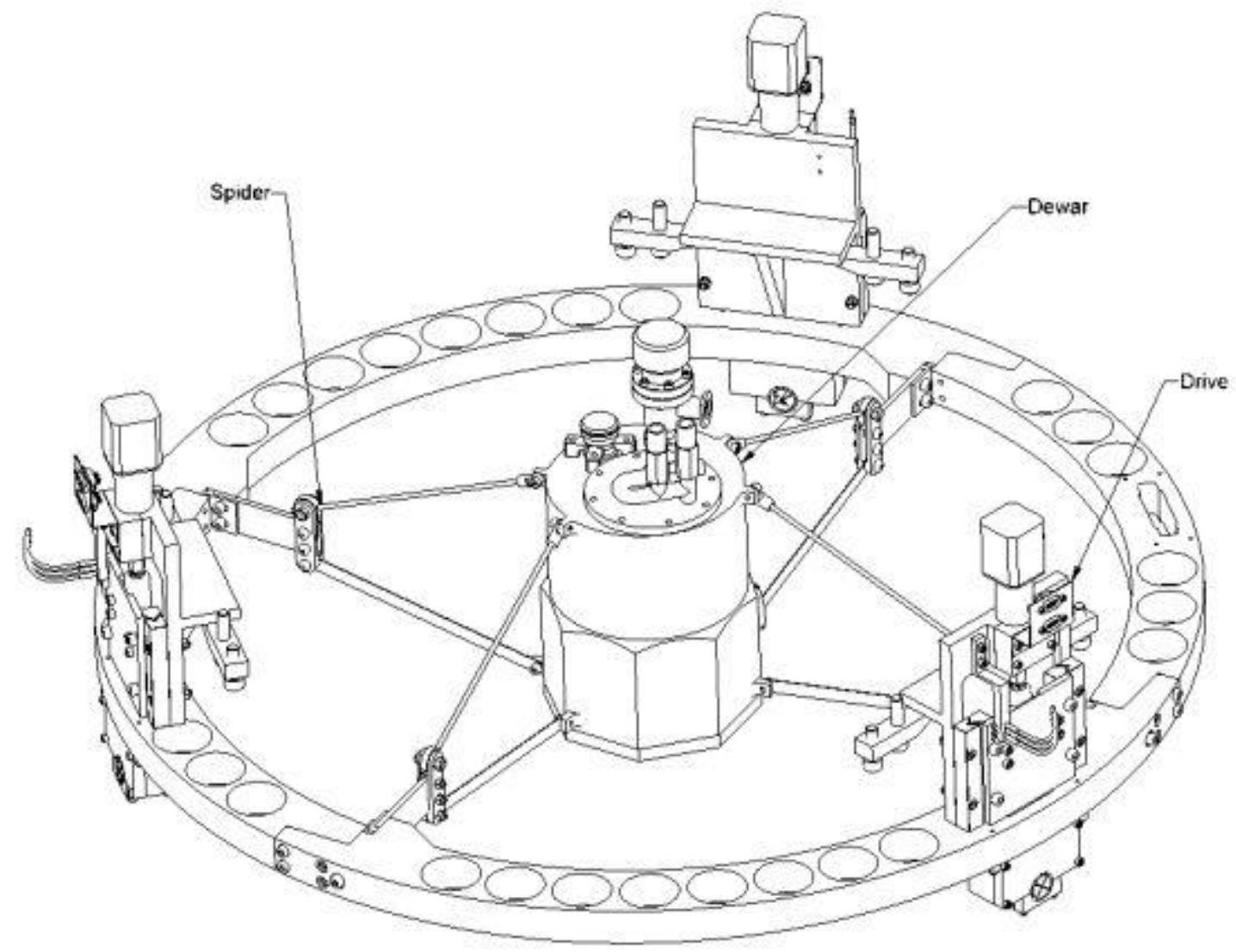

Fig. 6. View of the dewar mounted in a ring with three drives attached.
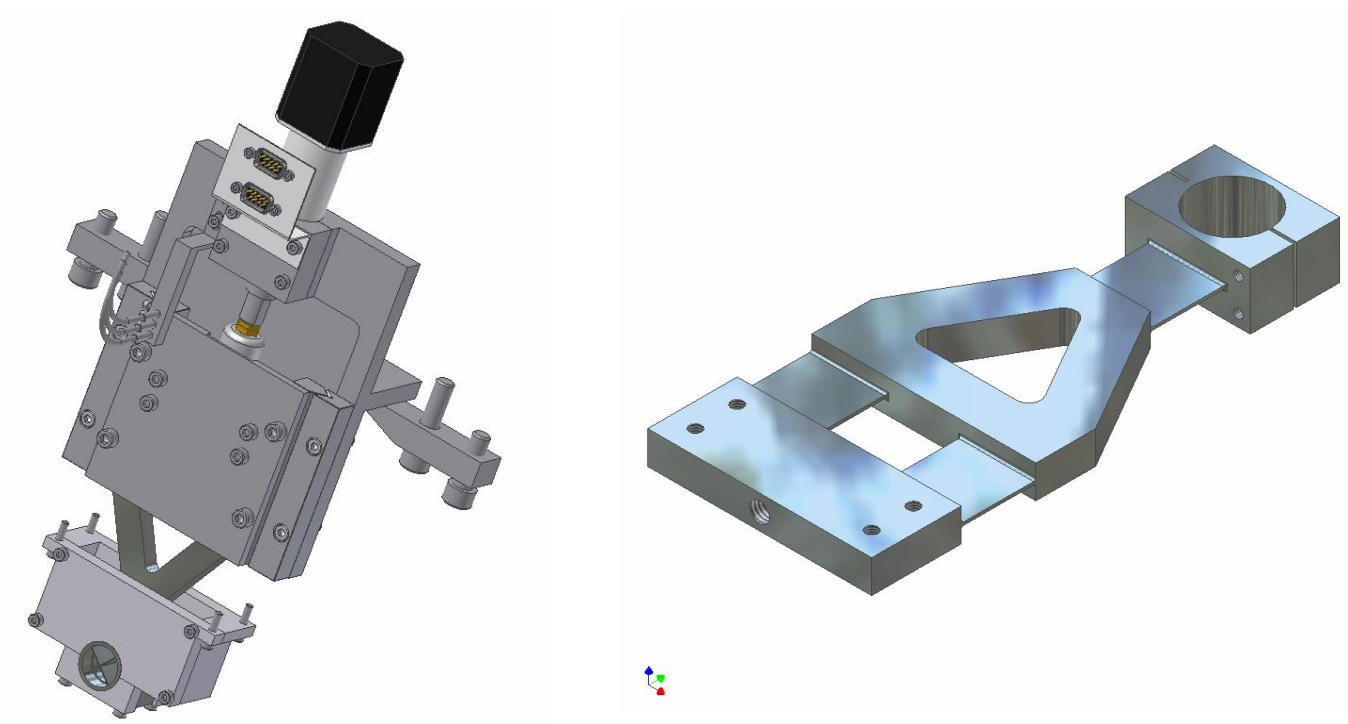

Fig.7. Tip, tilt, focus drive assembly.

Fig.8. Flex mount of the ring. 


\section{DETECTOR MOUNTING AND ALIGNMENT}

To date, instruments using mosaics of MITLL detectors have them mounted on individual molybdenum bases, which were then mounted to an aluminium frame. It was a sound method, but technologically rather difficult. It required specialized equipment for precision grinding of molybdenum, which itself is not a commonly available material. We have compared the heat transfer coefficient between molybdenum and aluminium nitride, which was used for the substrates of MITLL detectors as well as between aluminium and aluminium nitride. They were sufficiently similar. Our colleagues fom MSO (Mount Stromlo Observatory) who have more experience with testing of MITLL detectors have confirmed good results when using aluminium mounts in test dewars. Based on these findings we designed a mount (Figure 9) for two detectors machined from a single aluminium billet, to reduce the number of surface contacts in the thermal path. With the relatively large mass of aluminium we have achieved even heat distribution which allowed us (after confirmation by laboratory tests) to use a single heating element for both detectors thus simplifying control electronics.

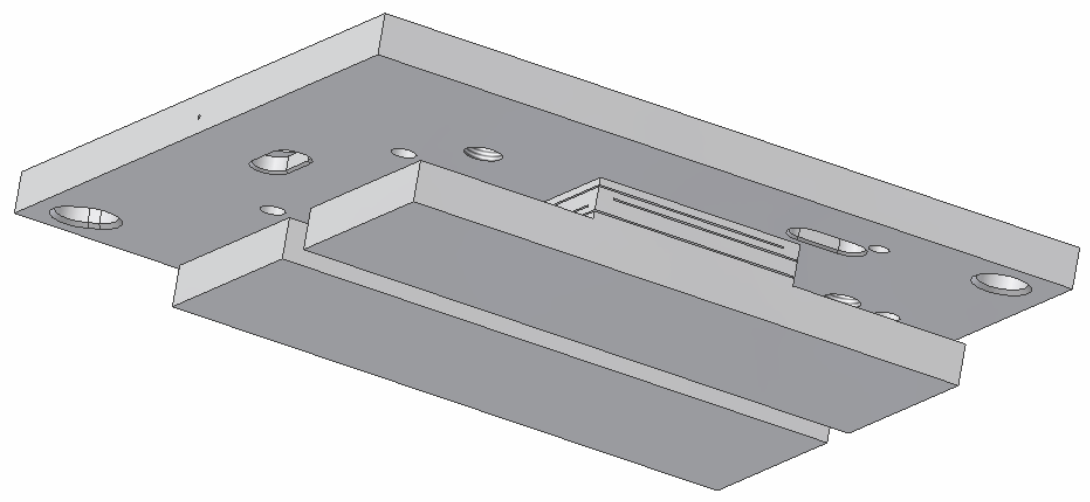

Fig.9. CCD mosaic mounting base.

For the best possible thermal contact with the under side of the CCD, the surfaces were lapped flat. The detectors' supports are formed on machined flexible stand-offs. These allow piston, tip and tilt adjustment with minimum stress.

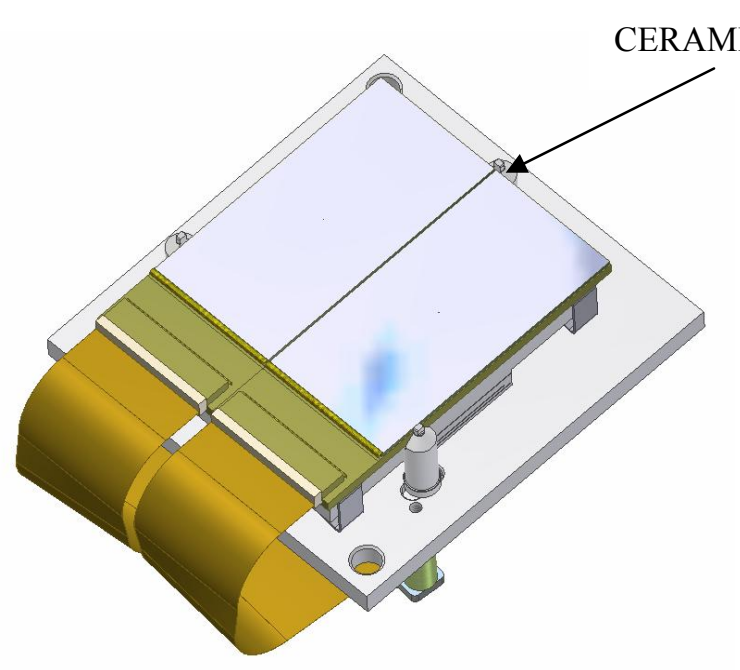

Fig.10. CCDs mounted on the base.

\section{SPACER PIN}
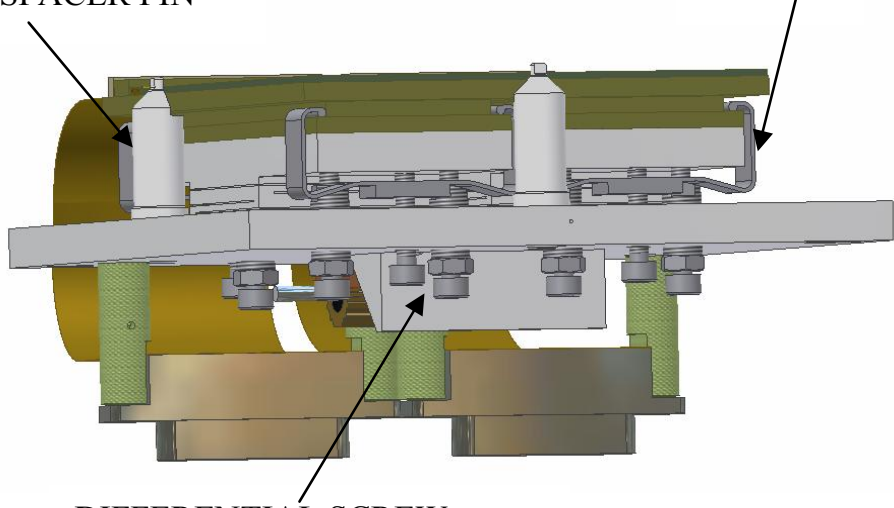

DIFFERENTIAL SCREW

The CCDs were held against the mount surfaces on mounting blocks with stainless steel clamps. The mounting blocks are adjustable against the base plate by means of stainless steel differential screws. The distance between the base plate and the field flattener is defined by three spacer pins machined out of ceramic material. The shrinkage of ceramics, steel 
and aluminium components was carefully calculated for the temperature after cool down and the distance between the surface defined by the three tips of the pins and the target position of the detectors' top surface for room temperature has been calculated. We have mounted the detector assembly on a stand with a space underneath to allow inserting a plate with spring loaded hexagonal rods suitable to engage with the differential screw heads. We then placed the stand with the detector and the plate with hexagonal keys engaged with the differential screws on the table of an optical measuring machine, a MicroVu® equipped with an Ealing Reflective Microscope Objective allowing measurements in the $z$ direction with close to 1 micron resolution. We then adjusted the stand's tilt with the use of shims until the small flat spots on the tips of the space pins did not show any deviation from the plane parallel to the table. Next we moved the measuring head into position focusing on the plane calculated for the position of the CCD faces. By taking multiple measurements and rotating the differential screws, we achieved a position where we could not detect any variation in the focus spot across the surfaces of the detectors. Finally we have checked the measurements to the tips of the space pins.

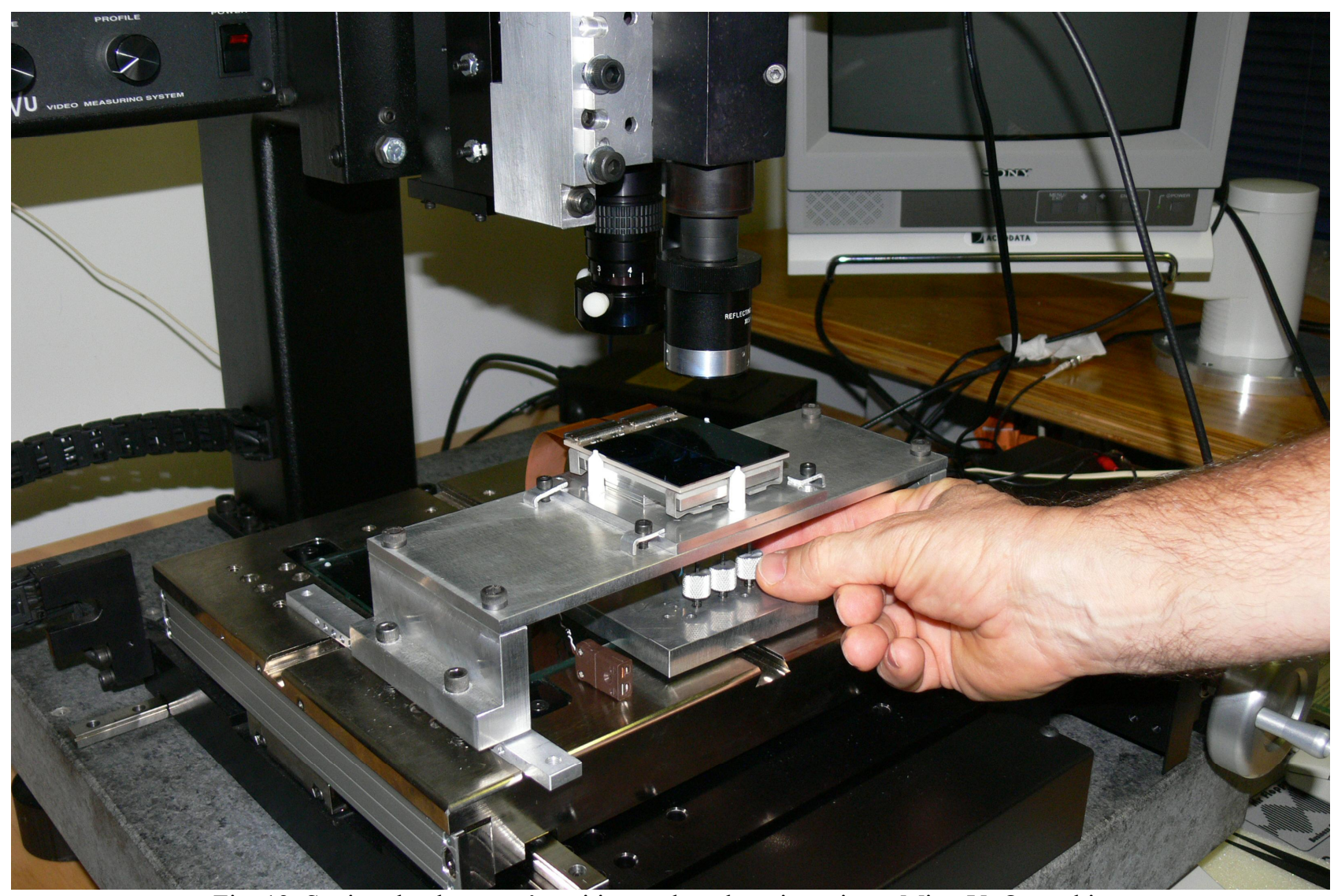

Fig. 12. Setting the detectors' position and coplanarity using a MicroVu® machine.

We adjusted the $x-y$ alignment of the CCD mosaic with the use of MicroVu® before the $z$ adjustment. We do not describe it here, as it was a relatively simple operation. 


\section{FIELD FLATTENER AS A DEWAR WINDOW}

A field flattening lens had to be installed as the detector dewar window since the filters had to be placed within a couple of millimeters of the front of the field flattener. As we have mentioned, the connectors of the detectors would have interfered with the lens, so we had to trim the lens to a square shape. The other reason for the square shape was confirmed with FEA: the edge of a circular lens would be too thin and the lens would flex under atmospheric pressure. There was only a room for a very narrow and thin support lip for the lens. A retaining frame could not be fitted due to lack of room, so the window had to be glued in. After our research and prototyping work, we chose a relatively simple but effective solution as shown in Figure 13.

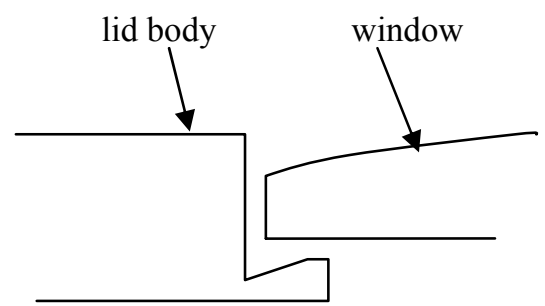

Fig. 13. The window mounting lip profile.

Figure 13 is a schematic diagram of the profile machined in the dewar lid. The small landing at the top right corner of the lip is a precision machined locator for the lens. There is a wedged groove towards the body of the lid. We have applied an accurately measured bead of cryogenic compatible flexible epoxy adhesive into the full length of the groove. Then we lowered the lens into the mount. As the lens was coming down, the proportions of the groove were changing and the adhesive was pushed up into the clearance between the lens and the lid body, rather than spilled into the lens. We practiced a few times with a flat window before the final attempt with the field flattener. We added three prongs to hold the lens down. This prevented eventual creeping of the adhesive to the optical surface of the lens before the glue was fully cured. There was also a safety aspect: we have used a Cryotiger ${ }^{\circledR}$ cooling system which contains compressed refrigerant; while we have used a safety valve on the dewar, the prongs on the window act as a secondary safety measure.

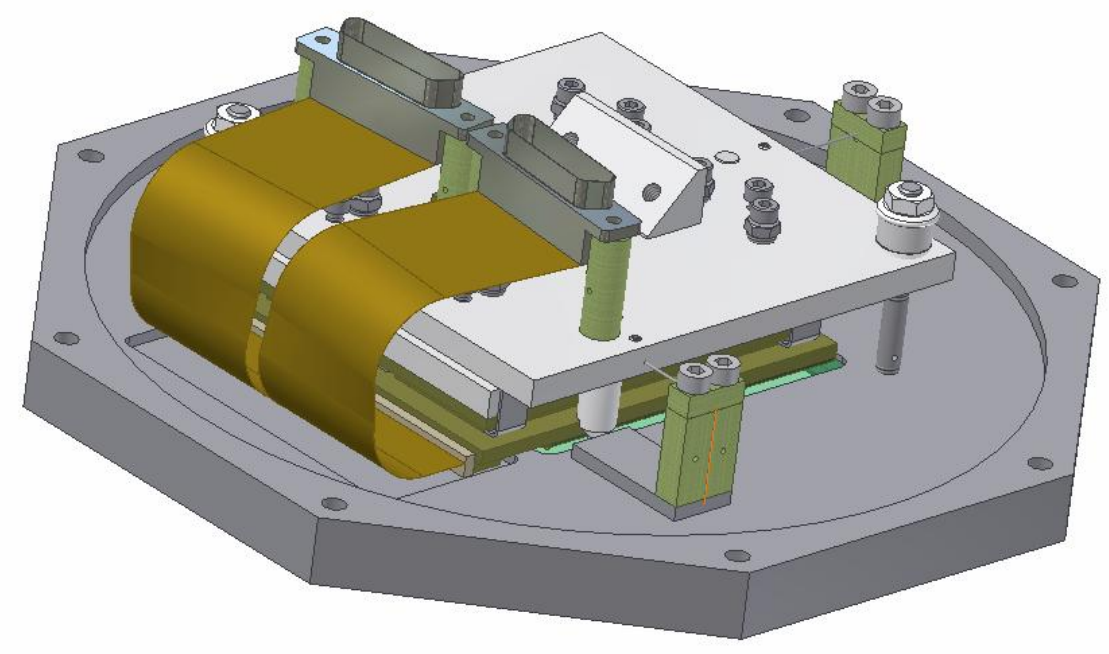

Fig. 14. The dewar lid with the window and detector assembly mounted. 


\section{CONCLUSION}

The physical constraints of putting a relatively large CCD mosaic into a Schmidt telescope with an $\mathrm{f} / 1$ beam, combined with a fast-acting robotic filter changer, required a considerable degree of engineering inventiveness. We hope that some of the ideas presented here may be useful to other instrumentation engineers.

The instrument described herein was delivered to the Automated Patrol Telescope in 2007, and is currently being commissioned.

\section{ACKNOWLEDGEMENTS}

This research was funded by the Australian Research Council, the University of New South Wales, the Australian National University, Swinburne University, and the Anglo-Australian Observatory. We are grateful for assistance from Chris Stubbs of Harvard University, Gerry Luppino of GL Scientific, Peter Conroy of MSSSO and Patrick Oats of MSSSO.

\section{REFERENCES}

[1] Carter, B. D., Ashley, M. C. B., Sun, Y-S, and Storey, J. W. V., "Redesigning a Baker-Nunn Camera for CCD Imaging", Proc. Astronomical Society of Australia, 10, 74-76 (1992). 